\title{
APROXIMACIÓN A LA VARIABILIDAD FRASEOLÓGICA DE LAS LENGUAS ALEMANA, INGLESA Y ESPAÑOLA
}

\author{
Gloria Corpas Pastor \\ Universidad de Málaga \\ gcorpas@uma.es \\ Florentrna Mena Martínez \\ Universidad de Murcia \\ flormena@um.es
}

\begin{abstract}
Resumen
Phraseological units, traditionally described by means of different properties, such as idiomaticity, fixedness and non-compositionality, are also subject to variation. Far from coming into conflict with the key phraseological characteristic (fixedness), variation reinforces its identity through various categories consistent across different phraseological types, different registers and different languages. This paper sets out to find a definition of phraseological variation and to establish a general framework of this phenomenon. We distinguish two main categories: variants and modifications and illustrate them with German, English and Spanish examples.
\end{abstract}

\section{Introducción}

Las primeras investigaciones lingüísticas que advierten la existencia de la fraseología ${ }^{b}$ destacan sobre todo las características que la distinguen y separan del resto de combinaciones de palabras potencialmente existentes en el sistema de la lengua, a saber, las combinaciones libres. Fruto de la comparación de ambos tipos de expresiones, fraseológicas y libres, surgen los rasgos de la fijación y la idiomaticidad que sirven para describir la naturaleza de las primeras. Tradicionalmente, pues, la fraseología se ha considerado como la parcela eminentemente estable de las lenguas, hasta el punto de merecer adjetivos como fosilizado, congelado, prefabricado, etc. La importancia del rasgo de la fijación es tan elevada e inherente a la identidad de las UFs (unidades fraseológicas) que la propia terminología hace uso

\footnotetext{
1 Entendemos por fraseología el conjunto de combinaciones estables formadas al menos por dos palabras gráficas y cuyo limite superior se sitúa en el nivel de la oración compuesta, las cuales se caracterizan por su alta frecuencia de (co)-aparición y su institucionalización en la lengua, así como los diversos grados de idiomaticidad y variación que éstas pueden presentar (Corpas Pastor, 1996: 269; Corpas Pastor, 1998a). El término fraseología es también la denominación que recibe la disciplina que estudia tales combinaciones.
} 
de dicho rasgo para nombrarlas. Sirva como ejemplo el título de la obra de Zuluaga (1980) Introducción al estudio de las expresiones fijas.

A estas alturas nadie duda en señalar la fijación como la primera propiedad esencial que debe poseer cualquier combinación de palabras que aspire a formar parte de la fraseología. No obstante, como es bien conocido, diversos estudios llevados a cabo han demostrado la existencia de un cierto grado de variabilidad que en principio podría poner en entredicho la naturaleza estable de las UFs. Además, la variabilidad dentro del universo fraseológico se presenta como un fenómeno complejo que acoge diferentes manifestaciones. Ante este panorama el objetivo de este trabajo es ofrecer una perspectiva general de la variabilidad en la fraseología que incluya una categorización de todas sus formas posibles, estableciendo en primer lugar la relación de este fenómeno con el rasgo de la fijación.

\section{Fijación y variabilidad: una relación de dèpendencia}

No es posible entender ciertos aspectos de la variabilidad en la fraseología sin mencionar la fijación y la relación de dependencia existente entre ambos conceptos. La fijación, rasgo fundamentalmente sintáctico para Ruiz Gurillo (1997), se define como la "propiedad que tienen ciertas expresiones de ser reproducidas en el hablar como combinaciones previamente hechas" (Zuluaga, 1975: 230). Se trata de una peculiaridad que aparece en algunas combinaciones de palabras cuando éstas son repetidas por los hablantes a lo largo del tiempo. Es, por tanto, un fenómeno diacrónico que además posee carácter gradual puesto que no todas las Ufs gozan del mismo grado de fijación. En las expresiones concretas es posible reconocerla a través de las diferentes huellas que imprime: el orden estable de los elementos integrantes, la estabilidad en las categorías gramaticales, la imposibilidad de suprimir, insertar o sustituir un componente, etc. La fijación posee, además, la capacidad de actuar a varios niveles lo que ha desencadenado la aparición de diversas tipologías. Así, por ejemplo, Thun (1978) distingue entre fijación interna, que se corresponde con los rasgos que acabamos de mencionar, y la fijación externa, dentro de la que es posible reconocer cuatro tipos: la situacional, la analítica, la pasemática y la posicional ${ }^{3}$.

Al margen de estas clasificaciones, el aspecto de la fijación que más nos interesa en este trabajo es el de las consecuencias que ésta acarrea. La estabilidad en la forma y la repetición pueden conducir a la institucionalización o convencionalización de las expresiones. Este efecto es tan importante que ha llevado a algunos autores como Baranov y Dobrovol'skij (1998: 29) a afirmar que "si falta la institucionalización, entonces no tiene sentido hablar de fijación desde el punto de vista de la estructura, ni desde cualquier otro". Además, la fijación supone para la combinación de palabras una menor capacidad de variación que el resto de expresiones libres de la lengua, lo que implica la posibilidad de predecir los componentes de la unidad lingüística, aspecto imprescindible y necesario para la generación de una de las manifestaciones de la variabilidad: la modificación.

El rasgo de la fijación, descrito y fundamentado en la estabilidad y complejidad de forma, la invariabilidad de los componentes léxicos, la imposibilidad de conmutación de los

2 Otras obras que destacan en sus títulos la cualidad estable de las UFs son, entre muchas otras, Salvador y Piquer (2000) El discurs prefabricat. Estudis de fraseologia teórica i aplicada; Moon (1998) Fixed expressions and idioms in English; Martínez Marín (1990) 'Las expresiones fijas del español y las relaciones de antonimia', etc.

3 Veásc Corpas Pastor (1996: 23-24) sobre estos tipos de fijación. 
constituyentes, la defectividad transformativa, etc. ${ }^{4}$, permite en algunas ocasiones formas alternativas que trasgreden sus preceptos. Es decir, la variabilidad no ocurre 'a pesar de' la fijación, sino que se presenta como una propiedad derivada, precisamente, de la existencia de esta característica:

Trotz - oder gerade wegen - ihrer Vorgeformheit werden sprachliche Schematismen jedoch haufig variiert, und das jenseits der Realisierungsmöglichkeiten, die bei manchen von ihnen als "usuell" gelten und die als "Varianten" sanktioniert sind". (Sabban, 1998: 13)

La variabilidad se hace posible justamente porque las UFs tienen una forma estable y unas marcas bien definidas. No en vano, la definición que el $D R A E$ ofrece del verbo variar ('hacer que una cosa sea diferente en algo de lo que era antes') pone de manifiesto la necesidad de dejar una parte inalterada y de reconocer, aunque sólo sea mínimamente, la base sobre la que la variación ha actuado. En el caso de la fraseología, la fijación junto con la idiomaticidad son los dos rasgos esenciales y responsables de que parte de la combinación de palabras permanezca inalterada, lo que a su vez permite identificar la forma original de la que se ha partido. Así pues, es posible calificar la fijación y la variabilidad como dos categorías complementarias dentro de la fraseología (Burger et alii, 1982: 67).

Por tanto, aunque la estabilidad sea un ingrediente esencial para el logro de la variabilidad, hay que admitir que el concepto de fijación tal y como fue planteado en las primeras investigaciones queda en entredicho. Si en un principio las UFs se definieron como unidades estables y fijas que mostraban rechazo a cualquier alteración léxica, semántica y morfosintáctica, en vista de la existencia de cambios reales y potenciales, no cabe hablar de la fijación como una propiedad absoluta (Häusermann, 1977; Burger, 1998) sino como una cualidad relativa (Fleischer, 1982; Gläser, 1986; Wotjak, 1992; Corpas Pastor, 1996; Burger, 1998) y, nunca mejor dicho, variable.

\section{Concepto de variabilidad fraseológica y sus manifestaciones}

El problema de la variabilidad fraseológica ha sido el objeto de estudio de distintos investigadores, sobre todo alemanes (cfr. Barz, 1986, 1992; Burger, 1998; Sabban, 1998) y soviéticos $^{6}$ (cfr. Dobrovol'skij, 1988). Ahora bien, este tema suele aparecer dentro de obras dedicadas a la descripción más o menos general de las UFs o de una categoría fraseológica concreta. En otras ocasiones la literatura nos ofrece análisis pormenorizados de alguna de las manifestaciones particulares de la variabilidad. No obstante, cabe destacar el trabajo de Burger (2000), dedicado en exclusiva al tratamiento global de este fenómeno.

4 Ruiz Gurillo (1997) analiza cada uno de los niveles en los que se produce la fijación (fonético-fonológico, morfológico, sintáctico, léxico-semántico y pragmático) y detalla los distintos aspectos que contribuyen a consolidarla.

5 "A pesar de -o precisamente por- su fijación, los esquematismos lingtísticos con frecuencia presentan variación. Dicha variación va más allá de las posibilidades de realización, entre las que destacan las variaciones «usuales» y las denominadas «variantes»." (La traducción es nuestra).

6 Desgraciadamente, el difícil acceso a algunas de las obras soviéticas nos impide poder referimos a ellas de primera mano. 
La relevancia de la variabilidad en el sistema fraseológico queda demostrada desde el momento en el que ésta afecta a sus rasgos más inherentes, lo cual implica la necesidad de incluirla dentro de las definiciones como una característica más de las unidades que conforman el universo fraseológico. Nuestro concepto de variabilidad fraseológica se construye desde una perspectiva amplia que nos permite acoger diferentes realizaciones del mismo hecho lingüístico. Variabilidad fraseológica es, pues, un término general que hace referencia a cualquier cambio o alteración de carácter léxico, semántico, morfosintáctico o incluso pragmático que se produzca en las UFs, exceptuando las producciones erróneas (ocasionales y espontáneas) de los hablantes.

Los estudios de corpus extensos (cf. Moon, 1998) que investigan este fenómeno han advertido su recurrencia en cada una de las distintas categorías fraseológicas ${ }^{7}$, si bien es necesario observar que los resultados estadísticos obtenidos dependen siempre del concepto de variabilidad que se haya empleado y del espectro que ésta cubra. Una de las cuestiones porcentuales más interesantes es la que relaciona el tipo gramatical de UF con el grado de variabilidad, así como la que establece conexiones entre los géneros textuales y la frecuencia de aparición de la variabilidad. Con respecto a la primera cuestión, Moon (1998: 121) afirma que la variación es más frecuente en las locuciones verbales que en las adjetivas, nominales o preposicionales. Por otro lado, el género que más explota la potencialidad de cierto tipo de variabilidad es el periodístico. Hecho que se ha visto confirmado en otros muchos estudios como el de Wotjak (1992) y Sabban (1998).

La variabilidad ha sido también considerada como un universal fraseológico suficientemente probado tras los trabajos realizados en diversas lenguas -alemán, francés, español, inglés, italiano, ruso, finlandés, holandés, sueco, etc.- a las que se le van progresivamente uniendo otras más minoritarias como es el caso de la lengua de un grupo étnico de Ghana (Yankah, 2000). Esta uniformidad en la presencia de la variabilidad en las lenguas contrasta con la ausencia de homogeneidad que este rasgo adquiere en su análisis dentro del sistema fraseológico de una lengua en particular. En realidad, se trata de un fenómeno que da cabida a múltiples manifestaciones que resultan de diferentes motivaciones e intencionalidades así como de diversos mecanismos lingüísticos. Estas motivaciones, junto con las peculiaridades lingüísticas, son las que determinan en última instancia las diferentes categorías que se vislumbran dentro del concepto general de variabilidad. De esta forma, adoptando los criterios de la intencionalidad, de la causa originaria y de las precisiones lingüísticas podemos distinguir dos grandes grupos: variantes sistemáticas y usuales frente a las modificaciones ocasionales.

Cuando la causa de la alteración o variabilidad de una UF proviene de las motivaciones conscientes de los hablantes que persiguen de esta manera alguna finalidad, hablamos de modificaciones. Si por el contrario, los cambios que se perciben en la unidad en cuestión no son fruto de las ansias innovadoras de los hablantes, sino meramente la realización de las posibilidades que el sistema lingüístico en general y el fraseológico en concreto ofrecen, estamos ante variantes institucionalizadas. Las modificaciones y las variantes emplean los mismos procedimientos en su generación pero difieren en las cuestiones pragmáticas, estilís- 
ticas y sociolingüísticas derivadas de la motivación del hablante, de los efectos ocasionados y de la institucionalización.

Además de estas dos manifestaciones de la variabilidad, conviene insistir en que un número considerable de UFs, por su propia naturaleza, invitan a la variación de sus elementos constitutivos. Por ejemplo, las unidades que presentan casillas vacías son buen exponente de la variabilidad como rasgo constituyente. Unidades fraseológicas como (al.) jmdm. fällt' rutscht das Herz in die Hose ('jmd. bekommt Angst', LDR), bei jmdm. einen Stein im Brett haben ('jmds Sympathien haben', LDR); (ing.) lick sb.'s boots ('coll. To behave in a slavish manner to a person in power, in an effort to impress him', LDOEI), twist sb. round one's little finger ('coll. To have the ability to persuade (a person) to do exactly as one wants', LDOEI); y (esp.) meter a alg. en cintura, caérsele a alg. la cara de vergüenza necesitan actualizar determinados actantes (sujeto, objeto, complemento) en el discurso para realizar su carga semántica. Esto es, las UFs con casillas vacías tienden una mano a las unidades léxicas del sistema de la lengua, las cuales necesitan y con las cuales se combinan no sólo para poder funcionar en el discurso, sino incluso para dotarse a sí mismas de sentido pleno.

\subsection{Las variantes}

Al igual que el resto de unidades, las UFs no aparecen en la lengua de forma aislada, sino en conexión con otras combinaciones fraseológicas a través de diferentes relaciones lingüísticas. Por ello, no sorprende que junto a una determinada UF aparezcan en una misma lengua otras, denominadas variantes, con las que guarda una relación de alta semejanza formal, semántica, funcional o pragmática. A este respecto, conviene distinguir entre variantes fraseológicas y unidades fraseológicas independientes, pero relacionadas, cuya diferencia fundamental reside en el plano metafórico, como muy bien señala Sabban (1998: 81) con respecto a la sinonimia de las UFs:

Synonym sind zwei Phraseme, wenn sie eine gleiche bzw. änliche phraseologische Bedeutung haben, sich jedoch - anders als die "lexikalischen Varianten" - in ihren Bildbedeutungen deutlich voneinander unterschieden ${ }^{8}$.

Algunos ejemplos de UFs sinónimas en las tres lenguas que utilizamos para la ejemplificación del presente estudio son (al.) Eulen nach Athen tragen y Wasser in der Rhein bringen ('etwas Überflüssiges tun', DR); (ing.) bear a grudge against sb. y have a chip on one's shoulder ('coll. A feeling of anger or bitterness because one thinks that one is regarded by others as a person of little value or worth', LDOEI); y (esp.) ser más listo que el hambre y saber más que los ratones coloraos.

Lo mismo es aplicable también a la caracterización de UFs antónimas, las cuales poseen un significado fraseológico opuesto, al tiempo que difieren en lo concerniente al plano figurativo o metafórico. A modo de ilustración mencionamos las siguientes fórmulas rutinarias de asentimiento y recusación en los tres idiomas: (al.) Das kommt überhaupt nicht in 
Frage!/ Aber natürlich! ('Das ist (nicht) akzeptabel'); (ing.) I couldn't agree more/ Tell it to the marines! ('I agree with you'/'coll. Don't expect me to believe that!', LDOEI); (esp.) De mil amores/ De eso nada.

Por el contrario, las variantes son el resultado de ciertas operaciones de índole léxica o estructural que se han llevado a cabo en una determinada UF dando origen a formas institucionalizadas 9 que presentan "efectos semánticos y pragmáticos triviales" (Dobrovol'skij, 1999: 368). Las variantes representan la parcela de la variabilidad caracterizada por ser usual y por tanto opuesta a la ocasionalidad propia de las modificaciones. Una de las definiciones más acertadas es la que proporciona Barz (1992: 29):

Von phraseologischen Varianten ist dann zu sprechen, wenn mindestens zwei Phraseologismen gleiche oder ähnliche phraseologische Bedeutung haben und außerdem so viele formale Übereinstimmungen, daß sie als Realisationen ein und desselben Lexems identifiziert werden können ${ }^{10}$.

Dentro de las variantes es posible reconocer distintos grupos atendiendo al tipo de cambio que se ha producido y a sus resultados. Las taxonomías existentes a este respecto suelen especializarse en una categoría concreta de UF. Así por ejemplo, Burger et alii (1982) y Wotjak (1992) ilustran sus tipologías preferentemente con locuciones; Barz (1992), más selectiva, se ciñe a un subtipo: las locuciones verbales; mientras que Mellado Blanco (1997) restringe su clasificación a los somatismos. De hecho, estas taxonomías no son en principio aplicables de forma generalizada a otros tipos de UFs, lo cual indica la necesidad de estudios más concretos que determinen los mecanismos de variantes para cada una de las categorías fraseológicas.

Utilizando criterios formales podemos localizar cambios léxicos, sintácticos y estructurales que dan lugar a las variantes léxicas, variantes estructurales y variantes perspectivas; categorías todas ellas presentes en dos de las esferas fraseológicas: las locuciones y los enunciados fraseológicos ${ }^{11}$.

Las variantes léxicas son formas fraseológicas ya establecidas en el sistema de la lengua, y por tanto institucionalizadas, que se han originado por sustitución léxica de uno o más componentes. Los lexemas que intervienen en este proceso mantienen relaciones semánticas tales como la sinonimia (total o parcial), la antonimia o incluso la metonimia y su sustitución o intercambio provoca en las variantes resultantes una relación de antonimia o sinonimia por lo que es posible distinguir entre variantes léxicas sinonímicas y variantes léxicas antonímicas. Algunos ejemplos de enunciados fraseológicos que constituyen variantes

9 Adoptamos la definición de institucionalización propuesta por Zuluaga (1997: 17): "A institucionalización (Fernando, Ch./Flavell, R. 1981) é a adopción da constucción pola comunidade lingüística. Institucionalización significa, pois, difusión xeneralizada, uso común e corrente".

10 "Podemos hablar de variantes fraseológicas cuando existen al menos dos fraseologismos que presentan un significado fraseológico idéntico o parecido, y además coinciden en tantos aspectos formales que podrían ser identificados como realizaciones de un mismo y único lexema" (La traducción es nuestra).

11 En este trabajo nos centramos en las unidades de la seguna y tercera esfera por ser éstas las más proclives a experimentar cualquier tipo de variabilidad. No obstante, la variabilidad es un fenómeno también presente en las colocaciones tal y como demuestran los siguientes ejemplos de modificaciones: (al.) einen Besen spielen (cf. eine Rolle spielen); (ing.) tell us the whale truth (cf. tell the truth y whole truth); (esp.) bofetones al contado (cf. dinero al contado). 
sinónimas son (al.) Besser ein Spatz in der Hand, als eine Taube auf dem Dach/Lieber den Spatz in der Hand als die Taube auf dem Dach ('Es ist besser, sich mit dem zu begnügen, was man bekommen kann, als etw. Unsicheres anzustreben, DUW); (ing.) A watched pot never boils/A watched kettle never boils ('worrying and anxiety about a situation will not hasten its development', LDOEI); y (esp.), A caballo regalado no le mires el diente/A caballo regalado no le mires el dentado. Ejemplos de variantes sinónimas de locuciones son también las siguientes: (al.) zwei Fliegen mit einem Schlag treffen/zwei Fliegen mit einer Klappe schlagen ('Mit einer Handlung zwei Ziele zugleich erreichen', DR); (ing.) a skeleton in the closet/a skeleton in the cupboard ("something of which a family, group, etc., is ashamed and which it tries to keep secret', LDOEI); (esp.) dormir como un leño/dormir como un tronco.

Tal y como se puede advertir en los ejemplos anteriores, las variantes léxicas sinonímicas presentan una completa congruencia estructural, una identidad semántica casi completa y una identidad léxica parcial (Dobrovol'skij, 1988: 163). Dependiendo del tipo de lexema que participe en la conmutación es posible distinguir varios subgrupos ${ }^{12}$ a los que no hacemos referencia por falta de espacio. Las variantes léxicas antonímicas, por su parte, nacen de la alternancia de lexemas antónimos con especial preferencia por los adjetivos y los verbos, tal y como demuestran los ejemplos que siguen a continuación: enunciados fraseológicos, como (al.) Ehrlich währt am längsten/Der Ehrliche ist der Dumme's ('Mit Ehrlichkeit besteht man am besten', DUW/'Ehrlichkeit führt zu nichts'); (ing.) Big is beautiful/Small is beautiful; y (esp.) quien bien te quiere te hará llorar/quien bien te quiere te hará reir; y locuciones, del tipo (al.) etwas schwarz/rosig malen ("etwas sehr pessimistisch/optimistisch darstellen', RD); (ing.) lose heart/take heart ('to become discouraged/to be encouraged', LODEI); y (esp.) poner buena cara/poner mala cara.

Las variantes estructurales representan el segundo gran bloque de UFs institucionalizadas que difieren únicamente en aspectos morfosintácticos. El significado fraseológico de estas unidades permanece prácticamente intacto mientras que ciertos cambios en el número, en el orden de los constituyentes, en la forma de la negación y en la utilización de preposiciones, artículos y conjunciones se encargan de indicar la existencia de variantes. $\mathrm{Si}$ en el caso de las variantes léxicas, la variabilidad se apoyaba en el aspecto léxico de las UFs, en las variantes estructurales es la morfosintaxis la protagonista y la responsable de la alteración de las unidades. Lo cual no implica que la frontera entre unas y otras esté nítidamente dibujada ya que las sustituciones léxicas acarrean en muchas ocasiones cambios estructurales. Estos cambios dan también como resultado unidades sinónimas y antónimas. Ejemplos de las primeras son los siguientes: enunciados fraseológicos, como (al.) auf Biegen oder Brechen/mag es biegen oder brechen ('etwas unter allen Umständen, um jeden Preis tun', RD); (ing.) Take care of the pence and the pounds will take care of themselves/Take care of the pennies and the pounds will take care of themselves ("if one is careful in the way one handles smaller amounts of money one need have no worries at all about the larger amounts', LDOEI); (esp.) ¿Quién te ha dado vela en/para este entierro?/ Nadie te ha dado vela paralen este entierro; y locuciones, como (al.) nicht Fisch, nicht 
Fleich/weder Fisch noch Fleisch ('Nicht zu bestimmen, einzuordnen sein', RD), (ing.) out at elbow/elbows ('old-fash. (of a person) untidy and wearing worn out clothes', LDOEI), y (esp.) irse a/de picos pardos.

Las variantes estructurales antonímicas, a diferencia de las anteriores, dejan notar sus efectos de manera decisiva en el aspecto léxico-semántico de las combinaciones fraseológicas al tratarse sobre todo de cambios en el sentido de la frase -de afirmación a negación o viceversa-, y de inversiones de los constituyentes. Ejemplos de enunciados fraseológicos serían (al.) Das ist dein Bier!/Das ist nicht dein Bier! ('Das ist (nicht) deine Angelegenheit', RD) ${ }^{14}$; (ing.) All roads lead to Rome/All roads don't lead to Rome ('All ways or methods of fulfilling a specific intention (don't) end in the same result', LDOEI); y (esp.) Afortunado en el juego, desafortunado en el amor/Desafortunado en el juego, afortunado en el amor. Como en el caso anterior, también se dan variantes antónimas entre las UFs de la segunda esfera, como ilustran los siguientes ejemplos: (al.) mit dem Strom/gegen den Strom schwimmen ('Immer mit der Mehrheit der gleichen Meinung sein'/'Sich gegen die allgemeine Meinung stellen', RD); (ing.) have an axe to grind/have no axe to grind ('(not) to be trying to gain personal profit or advantage', LDOEI); y (esp.) quedarse con la copla/no quedarse con la copla.

Dentro de este apartado se incluyen también las variantes perspectivas que hacen referencia no sólo a todas aquellas unidades que describen un suceso desde perspectivas recíprocas, sino también a aquellas que presentan cambios aspectuales, de causatividad, en la resultabilidad, en el número o tipo de actantes, etc. Los efectos estructurales y semánticos son la consecuencia lógica de los cambios realizados en el constituyente verbal. Este tipo de variantes no es muy frecuente en las paremias, de ahí que las investigaciones realizadas acudan al prototipo de UF, las locuciones, para ilustrar este fenómeno de variabilidad sistemática. A continuación mostramos algunos ejemplos: (al.) eins auf Dach geben/eins aufs Dach bekommen ('Jmdm zurechtweisen, tadeln', 'Von jmdn zurechtgewiesen werden' DR); (ing.) The cat is out of the bag/Let the cat out of the bag ('Everybody knows the secret'/(coll. To make known sth. that was a secret'); (esp.) ponérsele a uno los pelos de punta/ponerle a alguien los pelos de punta.

\subsection{Las modificaciones}

Las modificaciones ocupan la parcela más inestable, pero también más creativa, de la variabilidad. Se trata de UFs en las que los hablantes han llevado a cabo cambios ocasionales o alteraciones creativas persiguiendo cierta finalidad y que resultan en expresiones novedosas, no usuales. Por esta razón, es posible hablar de producción en la reproducción. Así pues, la UFM (unidad fraseológica modificada) es una unidad en parte producida y en parte reproducida por el hablante, es decir, es el resultado de un acto creativo y voluntario dentro de los límites de un lenguaje relativamente fijo y establecido.

Tal es la importancia de este rasgo fraseológico potencial que algunos autores consideran necesario incluirlo dentro de la definición de fraseologismo: 
Bei den PL handelt es sich um systemhaft (relativ) stabile, polylexikalische Einheiten, die gekennzeichnet sind durch (vollständinge oder zumindest teilweise) Idiomatizität, durch Lexikalisierung und somit Reproduzierbarkeit sowie durch eine hohe, in der Rede aktualisierbare textbildende (kreative Verknüpfunfs-, Assoziations- und Modifikations-) Potenz, ein sehr reiches kommunikatives Potential ${ }^{15}$. (Wotjak, 1992: 3)

El adjetivo "ocasional" tan frecuentemente empleado en las descripciones de la modificación, especialmente a la hora de distinguirla de las variantes, no implica que nos encontremos ante fenómenos carentes de asiduidad. No en vano, Fleischer (1982: 217) afirma que las modificaciones constituyen usos fraseológicos muy habituales y típicos. Esta afirmación está avalada por los resultados estadísticos de diversas investigaciones entre las que destacan Cernyševa (1980: 101) y Wotjak (1992: 133).

Entre las causas que pueden dar lugar al origen de una UFM se encuentra la finalidad que subyace a su uso. Los hablantes las producen por diferentes motivos entre los que destaca la búsqueda de nuevas formas de expresión que posiblemente calificarán al referente de una manera distinta y mucho más gráfica que la expresión convencional (Barz, 1986: 322). Junto a la intencionalidad se encuentra la propia naturaleza de la UF que impulsa o anima a los hablantes a la realización de transgresiones creativas (Gréciano, 1982: 237) y que algunos autores han identificado bajo el concepto de "potencia creativa textual". A este respecto Fleischer (1982: 216) señala que la diferencia de la potencia creativa textual de las UFs frente a la de los lexemas simples reside en el carácter especial de éstas como unidades lingüísticas.

La variabilidad potencial de los componentes fraseológicos es una página importante de la dialéctica existente entre la estabilidad y la variación lingüística. En el caso concreto de las modificaciones, se trata además de una combinación de posibilidades lingüísticas sistemáticas y la propia actividad comunicativa (Fleischer, 1982). Sin embargo, cabe preguntarse si esta potencialidad creativa de las UFs tiene límites. En este sentido, Burger et alli (1982: 68) apelan a las diferentes posibilidades de contextualización para aceptar prácticamente todas las modificaciones, ya que según estos autores apenas existen cambios fraseológicos que no adquieran un sentido en un determinado contexto. No obstante, cabe recordar que es la base fraseológica la que determina si se pueden producir modificaciones y la forma en la que éstas se pueden llevar a cabo (Barz, 1986: 322), lo cual implica que no existe una libertad absoluta a la hora de elegir el tipo y el procedimiento de la modificación.

Las manipulaciones creativas de todo tipo de UF, incluidas las colocaciones (Corpas Pastor, 1998b), son especialmente frecuentes en los textos periodísticos. El periódico Sur nos proporciona un ejemplo en el que asistimos a la manipulación por sustitución de la paremia iQue viene el lobo!, donde la figura amenazadora del animal y sus connotaciones son transferidas a la Junta de Andalucía, visualizada como un peligroso enemigo potencial. Pero en este caso, estamos ante un uso irónico de la UF, en tanto se critica la actitud, supuestamente simplista e infantil, del alcalde de Málaga en su enfrentamiento con el Gobierno

15 "Los fraseolexemas constituyen unidades polilexemáticas del sistema con (relativa) estabilidad, que se caracterizan por la idiomaticidad (completa o al menos parcial), la lexicalización, la reproducibilidad, así como por un potencial comunicativo muy rico, es decir, por estar dotados de una potencia constructora de texto (potencia creativa de conexión, de asociación y modificación) que se actualiza en el discurso". (La traducción es nuestra) 
andaluz a cuenta de la próxima construcción de un metro en la ciudad de Málaga asumida por una institución.

Como en el viejo cuento infantil, de tanto gritar ique viene el lobo! Al final ese recurso pierde efecto. $\mathrm{Y}$ en el Ayuntamiento se ha abusado, hasta el exceso, del ;que viene la Junta!... Los ciudadanos, al final, saben distinguir el grano de la paja. Y el alcalde se ha encontrado con que el recurso no cuela. (Sur, 02-11-03: 19).

No obstante, las modificaciones no sólo utilizan la sustitución de constituyentes léxicos como mecanismo productivo. Este tipo de variabilidad adquiere diversas formas que dependen de varios elementos: la combinación fraseológica propiamente dicha, el contexto y la intencionalidad del hablante. Ante una determinada UF y un contexto concreto, la lengua pone a disposición de los hablantes distintos procedimientos de los que pueden hacer uso para lograr la finalidad que los impulsa a realizar la modificación. Muchas veces no se puede hablar de modificaciones en cuanto a la integridad de los elementos constituyentes de una determinada UF, sino más bien de reliteralizaciones, de prominencia o simultaneidad de planos figurativos y literales, de actualizaciones zeugmáticas de sentidos, etc. propiciados por la simple yuxtaposición de unidades, ya sean fraseológicas o no.

En el siguiente ejemplo, extraído del mismo número del periódico Sur, titulado "Mar adentro", entran en juego unidades como hacer deporte y hacer de tripas corazón, junto a tripas; y pasarlo en grande, junto a pasar. El efecto creativo se consigue, precisamente, a través de las cadenas léxicas cohesivas, literales (windsurfistas, olas, playa, mar, pateras, equipos de rescate), y sus efectos sobre los componentes individuales de dichas UFs, las cuales no sólo actualizan sus significados unitarios, traslaticios o delexicalizados, sino también, y al mismo tiempo, sus significados individuales (compositivos y lexicalizados).

Los equipos de rescate no dan abasto y en Cádiz se juntan en la playa los cadáveres y los windsurfistas. Los que hacen deporte y los que hicieron de tripas corazón y se embarcaron en pateras porque tenían las tripas vacías. Las olas de dos metros se portaron desigualmente con ambos grupos: unos lo pasaron en grande y otros no pudieron pasar. (Sur, 02-11-03)

También hay que decir que la presencia simultánea de varios procedimientos de modificación es una constante en el discurso. A modo de ejemplo, reproducimos un fragmento procedente de un artículo de opinión titulado "Midnight Oil Provides Too Little Light", firmado por Christine Pullar para una publicación periódica destinada a especialistas en biotecnología ${ }^{16}$ :

I think that you can come on to a campus at almost any hour night or day and find some poor postdoc working away diligently, burning the proverbial candle at both ends, but the supervisor will probably be unaware. How do you prove to your boss that you spend the required hours in the lab without having to stamp a time card? You can't. (The Scientist, 2003, 17[19]: 49)

$16 Y$ es que, como mantenemos en este trabajo, la variabilidad, incluso la más creativa y transgresora, no sólo se encuentra en la lengua corriente, en la prensa diaria o en los usos eminentemente literarios, sino que se trata de una cualidad de la fraseología que se observa, incluso, dentro del discurso científico especializado. 
La autora narra su experiencia personal como investigadora en una universidad americana. En su opinión, los investigadores posdoctorales pasan largas jornadas de trabajo en el campus, al cual acceden de día y de noche. Sin embargo, los directores de los proyectos para los cuales trabajan no siempre aprecian todo el tiempo que éstos pasan en los laboratorios. De ahí que la autora concluya que, a la postre, compensa dedicar menos horas al trabajo y tener una vida fuera de la Universidad. Al estar más descansados y cargados de energía, se trabaja con más calidad y en plenitud de facultades. Para apoyar su argumentación, la autora emplea dos UFs manipuladas creativamente mediante la reducción de elementos integrantes ("midnight oil", alude a la paremia entera) y la adición de unidades léxicas ("burning the proverbial candle at both ends", donde el adjetivo proverbial subraya el aspecto de sanción social, cercano al valor de verdad general del que gozan la mayor parte de las paremias). Ambas UFs comparten en sus formas canónicas un mismo constituyente, burn: burn the midnight oil ('not fml. to work very late, esp. to study', LDOEI) y burn the candle at both ends ('colloq. to lead a busy life during the day and also go to bed late at night, esp. in order to have a full social life', LDOEI), y refuerzan la idea del trabajo (sobre todo intelectual), la división natural entre actividades diurnas y nocturnas, así como el equilibrio/desequilibrio entre la vida profesional y la vida privada (cf. la paremia All work and no play make Jack a dull boy, que preconiza la necesidad, reconocida socialmente, que tiene el ser humano de encontrar un equilibro en la vida entre las facetas profesional y privada).

Llegados a este punto, conviene señalar que una determinada UF no está restringida a un determinado procedimiento de modificación, como tampoco existe una clara relación de biunivocidad entre el tipo de manipulación empleada y el efecto conseguido. Por ejemplo, la UF alemana Wer A sagt, muss auch B sagen ('Wer etwas beginnt, muß es fortsetzen und auch unangenehme Folgen auf sich nehmen', DUW) - esto es, hay que terminar lo que se empieza, o bien, si se admite o dice una cosa, entonces hay que admitir o decir también otra cosa- presenta sustitución antonímica de elementos de la cláusula principal en la reformulación de Bertold Brecht: "Wer A sagt, muss nicht B sagen. Er kann auch erkennen, dass A falsch war.", es decir, si se dice $\mathrm{A}$, no se ha de decir B, porque pudiera darse el caso de que también se estuviera reconociendo que A es falso. Esto es, la nueva UF rechaza la interpretación estándar de otra UF, anterior a ella, al tiempo que lo hace con la fuerza que le confiere el haberse "apropiado" de las connotaciones y significado fraseológico de ésta, precisamente para refutarla.

El siguiente ejemplo muestra la manipulación creativa de la unidad que nos ocupa en el discurso. Pero ahora los procedimientos empleados son la adición de elementos que sirven de anclaje de la UF en su co-texto, al tiempo que se especifica el contenido abstracto de A, que en este caso hace referencia a las funciones circenses, y B, que a su vez se refiere a las representaciones teatrales. En este caso, pues, no se refuta la interpretación estándar, sino que se particulariza la referencia abstracta e indeterminada de A y B, aplicándolas al circo y a teatro, reforzando además la idea de que ambas son manifestaciones de una misma clase de actividad "artística".

Es wäre unfair, dem Öz einen Vorwurf daraus zu machen, daß [sic] er sich für seine Ausländer einsetzt. So ist das nun mal. Wer A sagt zum Zirkus, der muß auch B sagen zur Vorstellung. (URL: http://www.politikforum.de/forum/) ${ }^{17}$.

17 Todas las direcciones URL mencionadas en el presente estudio se encontraban operativas en la fecha de consulta (14-11-03). 


\subsubsection{Procedimientos de modificación}

La clasificación de todos los procedimientos existentes ha dado lugar a diferentes categorías. Algunos fraseólogos, siguiendo a Koller (1977) distinguen entre modificaciones internas, caracterizadas por ser cambios que influyen visible y directamente en la estructura formal de la UF, y modificaciones externas que hacen referencia a las alteraciones que dejan dicha estructura intacta concentrándose exclusivamente en el aspecto semántico de la expresión. Otros autores (Burger, 1998) piensan que esta clasificación es poco práctica ya que existen dificultades a la hora de ubicar en uno $\mathrm{u}$ otro grupo ciertos procedimientos como el de la separación. En su opinión, la dicotomía interna-externa no refleja con suficiente claridad las diferencias existentes entre unas clases y otras, ya que es el contexto el que en última instancia decide si la semántica de una UF ha sido afectada independientemente de la existencia de cambios formales. Burger (1998) propone por ello una primera división entre:

- Modificaciones que afectan a la forma exterior, a la parte externa de la UF, es decir, a la distribución de sus constituyentes, a sus componentes léxicos y a su estructura morfosintáctica.

- Modificaciones que sólo refieren al significado de la UF, sin que su forma externa se altere.

El primer tipo de modificación puede tener consecuencias semánticas o no tenerlas, por lo que la clasificación se perfila incluyendo tres grupos:

- Modificación formal sin modificación semántica

- Modificación formal con modificación semántica

- Modificación semántica sin modificación formal

Las características de las UFs influyen decisivamente en el establecimiento de los tipos de modificación que manifiestan. Es cierto que las UFs se caracterizan por su polilexicalidad, por su fijación e idiomaticidad, pero también es cierto que el sistema fraseológico está poblado por unidades que difieren considerablemente en cuanto al grado de fijación e idiomaticidad que presentan. Es más, algunas exhiben distintos tipos de fijación y, sobre todo, distintas condiciones contextuales y pragmáticas. Todo ello hace necesaria la elaboración de una clasificación general que acoja todo tipo de alteraciones creativas sin menoscabo de que algunas unidades sean más propensas a manifestar ciertos procedimientos modificadores en lugar de otros. Por esta razón, partimos de una primera división que pretende deslindar aquellos cambios no estandarizados que se producen en el interior de la estructura formal de la combinación fraseológica, de aquellos otros cambios que tienen que ver con sus condiciones semánticas, contextuales y pragmáticas:

- Modificaciones formales

- Modificaciones semántico-contextuales y pragmáticas

Esta distribución muestra muchas similitudes con la propuesta por Koller, pero sobre todo coincide con la de Burger en su intento de separar las variaciones que ocurren en el 
aspecto formal de las UFs de las que se producen en relación con otros aspectos que conectan la unidad con su entorno. Sin embargo, difiere de ambas al intentar separar los cambios de los efectos semánticos que éstos producen. Las alteraciones ocurren bien en la estructura formal, o bien al poner en contacto la unidad con un contexto pragmático y textual que le es ajeno. Todas y cada una de estas alteraciones pueden tener consecuencias semánticas, pero dichas consecuencias no constituyen en sí mismas modificaciones sino que forman parte de los efectos que éstas ocasionan y del proceso creativo que se desencadena conocido como desautomatización ${ }^{18}$.

\subsubsection{Modificaciones formales}

Las modificaciones formales hacen referencia a cualquier manipulación intencionada y creativa que se produzca en la estructura formal de las UFs. Puesto que este tipo de modificación puede causar cambios semánticos es conveniente distinguir, tal y como lo hiciera Burger (1998), entre modificaciones formales sin cambios semánticos y modificaciones formales con cambios semánticos. El primer grupo es el más marginal por el hecho de contar con una escasa cantidad de ejemplos ya que es difícil encontrar una UF alterada formalmente sin que ello tenga consecuencias en el plano semántico. No obstante, los procedimientos que pueden encajar en este marco en algunas ocasiones son la ampliación léxica y sintáctica por medio de algún adjetivo o la reducción de alguno de los componentes.

Normalmente, la manipulación de cualquier rasgo formal en el seno de una UF tiene consecuencias directas en su significado por lo que junto al cambio de estructura también se ocasionan efectos semánticos. Teniendo en cuenta el procedimiento empleado en la variación de la estructura externa de la UF podemos distinguir entre: sustitución léxica, extensión, reducción, modificación gramatical y fusión o contaminación. Al margen de todos estos grupos, es necesario advertir que con frecuencia las UFMs presentan más de un procedimiento de ruptura creativa.

La sustitución léxica designa el procedimiento mediante el cual uno o más elementos de una UF son reemplazados por otros lexemas. El éxito se intenta garantizar mediante diferentes mecanismos pensados para la correcta recepción y comprensión de la UFM y basados frecuentemente en relaciones semánticas y paronímicas entre los constituyentes implicados o en una relación contextual que se establece entre el nuevo lexema y el contexto en el que se produce la modificación. Por ejemplo, el primer fragmento procede de una sección firmada por Jeannette Lofas dentro de la publicación electrónica Lovegevity <URL: http: //www.lovegevity.com/parenting/parentingadvice/familyrules $2 . h t m l>$.

La autora comenta la situación de la institución matrimonial en Estados Unidos a partir de los datos estadísticos ofrecidos por el Instituto de Estadística americano. En el título utiliza una UFM, donde se sustituye el elemento de la UF institucionalizada (Actions) por uno nuevo (Numbers) para acentuar la veracidad de los datos estadísticos como forma de abordar esta realidad, frente a otras formas posibles de tratar este tema candente de la sociedad norteamericana (comentarios impresionistas, opiniones personales, posturas oficiales de organizaciones religiosas, etc.). 
Numbers Speak Louder Than Words

As the following statistics demonstrate; we have definitely become a Family 2000 and step-relationship society:

1. The fastest growing marital-status category is divorced persons.

2. By the year 2000 , more Americans will be living in stepfamilies than in nuclear families. (U.S. Census Bureau, 1990)

3. When we include living-together arrangements in our definition of "family," close to 50 percent of all women, not just mothers, will at some time live in stepfamily relationships. (Professor Larry L. Bumpass, University of Wisconsin, 1994)

4. One out of two marriages ends in divorce. (U.S. Census Bureau, 1990)

En el siguiente ejemplo, el nombre del actual presidente norteamericano sustituye a Dios en la fórmula de despedida Hasta mañana, si Dios quiere. Se trata de un artículo aparecido en la sección "Crónica de León", del periódico El Mundo, que versa sobre las mobilizaciones en el Bierzo contra la intervención militar estadounidense en Irak. En este caso, el procedimiento de la sustitución produce desautomatización por actualización de los dos planos (figurativo y literal), al tiempo que se critica con ironía los delirios de grandeza y la megalomanía de Bush.

\section{¡HASTA MAÑANA... SI BUSH QUIERE!}

Estamos perdidos. La razón ha perdido la razón. Mientras Dios está presente en las palabras de Bush, que pide a los americanos que se levanten y recen con él "en este tiempo de prueba", en Irak hay niños, mujeres y hombres que amanecen bajo la amenaza del terror. Inocentes que quizá mañana no puedan celebrar la alegría, el gozo de vivir, porque a los americanos "Dios les enseñará a tener resolución a la hora de enfrentarse contra el mal". (El Mundo"19, 15-02-03).

La sustitución semántica, denominada por Barz (1986) paradigmática, utiliza el significado de los elementos integrantes de la UF originaria para lograr sus objetivos. Las relaciones que se establecen entre el componente sustituido y el sustituyente son la sinonimia, la antonimia, la hiperonimia, la hiponimia y la cohiponimia, entre otras. Ejemplos de este último tipo son los siguientes: An onion a day keeps everybody away (cf. An apple a day keeps the doctor away) y Ojos que no ven, estómago que no siente (cf. Ojos que no ven, corazón que no siente). Estos enunciados ilustran cómo la variación creativa hace uso del plano semántico de los constituyentes que intervienen en el proceso para la elección de los nuevos lexemas.

La sustitución paronímica emplea el significante de dichos componentes apoyándose en la similitud fonético-fonológica para construir la modificación. El siguiente ejemplo ha sido extraído del listado de antiproverbios (anti-proverbs) que ofrecen Mieder y Litovkina (2002): Taste makes waist, esto es, la buena mesa hace engordar, a partir de la paremia inglesa Haste makes waste ('Hurrying will actually slow things down', TW).

En otros casos, la modificación se sustenta en el reordenamiento de los elementos integrantes de la unidad, como se observa en el siguiente fragmento, sobre la base de la UF alemana Vertrauen ist gut, Kontrolle ist besser ('Man soll sich nur auf das verlassen, was 
man nachgeprüft hat', DGW). Dicha unidad aparece modificada en el título de un artículo de opinión que firma Florian Rötzer, y que reza así:

\begin{abstract}
Kontrolle ist gut, Vertrauen ist besser.
Elektronische Überwachung von Computerarbeitsplätzen steigert nach einer Studie nicht die Leistung, sondern führt nur zur Anpassung an das unbedingt Notwendige. Wenn schon die technischen Möglichkeiten vorhanden sind, so könnte auf den ersten Blick die Idee vernünftig erscheinen, zur Förderung der Produktivität die Angestellten auf Schritt und Tastendruck zu überwachen. Das empfehlen natürlich die Unternehmen, die die entsprechende Software herstellen, um beispielsweise die Arbeit der Angestellten am Computer und deren Benutzung des Internet zu kontrollieren (Telepolis, 06-12-2001: 10)
\end{abstract}

En dicho artículo el autor argumenta que la excesiva vigilancia en el trabajo, utilizando para ello incluso medios electrónicos (ordenadores, monitores de videovigilancia, etc.) para observar al personal, no se traduce precisamente en un aumento de la productividad, sino más bien en todo lo contrario. En otras palabras, si la UF en su forma canónica recomienda ser algo desconfiado, la UFM hace referencia a la necesidad de dar a los trabajadores el margen necesario de confianza para que se sientan cómodos y relajados. Obsérvese que en ese mismo párrafo aparece también otra UFM "auf Schritt und Tastendruck zu überwachen", sobre la base de la locución jdm. auf Schritt und Tritt folgen ( $\mathrm{j}$-m überallhin folgen', LGDAF), donde se ha utilizado el procedimiento de la sustitución: folgen (seguir)/überwachen (vigilar) y Tritt (paso)/Tastendruck (golpe, pulsación): se acentúa, por un lado, lo detallado del seguimiento (überwachen), que se convierte más bien en una vigilancia férrea en previsión de que el trabajador no estuviese desarrollando correctamente sus tareas o bien otras, no previstas y de algún modo, prohibidas desde la dirección de la empresa; y por otro lado, se enfatiza el empleo de medios tecnológicos (Tastendruck) para seguir muy de cerca todos los movimientos del trabajador.

Los siguientes ejemplos nos sirven para ilustrar los procedimientos de extensión, reducción y modificación gramatical. En el primer ejemplo se inserta la secuencia ear wax en la UF institucionalizada, crumbs from the rich's man table ("Things that remain after a rich man has finished, and which a poor person can have', ODOEI).

It's because I feel superior. There he is, poor buger, picking up the odd crumb of ear wax from the rich's man table. Your're right. I don't mind. I like it. I like the way his presumption admits his poverty. (en Corpas Pastor, 1994: 438)

El segundo fragmento ilustra el empleo de la UF El gato escaldado del agua fría huye, reducida a la secuencia "gato escaldado", que, en calidad de núcleo residual, evoca, con efecto, la unidad completa. En el fragmento se hace referencia a las declaraciones de Jordi Sevilla, responsable de Asuntos Económicos del PSOE, sobre un posible gobierno con el PP y la actitud que piensa adoptar con respecto a una supuesta situación de corrupción económica (actitud que deriva de sus experiencias previas en casos de corrupción).

El responsable socialista de economía sitúa como punto de despegue de su partido las próximas elecciones municipales y autonómicas y advierte del estilo bronco y de "acoso y derribo contra el PSOE", adoptado por un José María Aznar que pretende -según Sevilla- expiar el daño que ha causado al PP convirtiéndose en el protagonista de la campaña. 
Hablando de un hipotético gobierno del PSOE, Jordi Sevilla asegura que huirá "como gato escaldado" de la corrupción y que pondrá negro sobre blanco las ahora "oscuras" [sic] y "creativas" cuentas públicas. (El Siglo $\left.{ }^{20}, 05-05-03\right)$

En el siguiente ejemplo asistimos a la modificación gramatical de la UF Crime does not pay ('criminal behaviour is not only wrong, but foolish from a practical point of view', ODOEI) de forma que se expresa una interpretación estándar opuesta. Esto es, según una noticia difundida por la BBC en una de sus publicaciones digitales, un porcentaje muy alto de jóvenes británicos considera que 'el delito compensa'. Tan sólo les detiene el temor de verse entre rejas.

Four out of 10 teens think 'crime pays'.

Four out of 10 teenagers in the UK think that crime pays, and in poorer areas that figure jumps to six out of 10 . A study of 600 children aged between 13 and 19 also said that prison was the biggest reason why they wouldn't commit a crime. (BBCl. Newsround. 16-09-03) <http://news.bbc.co.uk/cbbcnews/>

Los sustantivos que designan todas las categorías modificadoras mencionadas son muy explícitos y describen a la perfección los cambios a los que hacen referencia. Así, la extensión acoge la variación ocasional consistente en añadir más constituyentes a la UF originaria, la reducción opera de forma contraria sustrayendo componentes, mientras que la modificación gramatical actúa como epígrafe general bajo el que se reúnen diferentes alteraciones como la inversión de elementos, el cambio de afirmación a negación, el cambio en el número de algún constituyente, etc. Junto a los anteriores, los términos de fusión o contaminación, ligeramente más opacos que el resto, designan el procedimiento de modificación que se basa en la mezcla de dos o más UFs (Palm, 1995: 73), como, por ejemplo, (al.) Aus der Reihe fallen (cf. aus der Reihe tanzen + aus dem Rahmen fallen); (ing.) You are my tower of Gibraltar (cf. You are a tower of strength + You are my rock of Gibraltar ${ }^{2 \mathrm{i}}$ ); o (esp.) Fue a por lana y recibió tres tazas (cf. ir a por lana y salir trasquilado + Si no quieres caldo, toma tres tazas), Cría cuervos y échate a dormir (cf. Cría cuervos y te sacarán los ojos + Cría fama y échate a dormir) y En el país de los ciegos, rey tuerto, rey puesto (cf. En el país de los ciegos, el tuerto es el rey $+A$ rey muerto, rey puesto), ejemplos estos últimos extraídos del refranero impopular, recogido por José R. Brox (<http://www.snarkianos.com/ recopilaciones $15 . \mathrm{html}>$ ).

\subsubsection{Modificaciones semántico-contextuales y pragmáticas}

En este apartado se incluyen todas las modificaciones que a pesar de afectar de forma contundente a la UF no ocurren en su interior sino en su entorno textual o pragmático. Puesto que no afectan a la estructura interna y formal de la combinación son imperceptibles a simple vista sino se presentan junto a su contexto. Gracias a los potentes efectos que desencadenan, estas modificaciones consiguen ensalzar y aprovechar la creatividad de las 
UFs mediante la violación de alguna de sus condiciones de selección, por lo que es posible distinguir entre modificaciones producidas por la violación de las condiciones semánticas de selección, por la violación de las condiciones lingüístico-textuales, y por la violación de las condiciones pragmáticas.

Ilustraremos este tipo de modificación con algunos ejemplos en las lenguas objeto de nuestro estudio. El fragmento que reproducimos a continuación procede de un artículo de opinión sobre una demanda judicial interpuesta por un trabajador británico contra su empresa por un supuesto delito de discriminación sexual. El demandante argumenta que la empresa le obliga a usar corbata en su puesto de trabajo, mientras que a las trabajadoras mujeres no les obliga a llevar prendas incómodas. El efecto creativo proviene de la yuxtaposición del refrán, introducido, además, por el presentador "There's that old saying", que automáticamente desencadena la interpretación metafórica de la UF, y una secuencia homónima literal, a la cual se añade otra secuencia literal que refuerza la interpretación de los significados literales e individuales de los componentes de la UF institucionalizada, pero dentro de la interpretación estándar de ésta, la cual refuta: el hecho de llevar corbata no te hace más profesional, esto es, la indumentaria es indiferente, pues, el hábito no hace al monje.

There's that old saying, "clothes make the man." What most people don't realize is that this is only part of the old saying. The complete old saying is "clothes make the man feel like he is being slowly asphyxiated." My guess is that the old saying was never completed because the person who wrote it was wearing a necktie and passed out from oxygen deprivation before he could finish his thought. But it is unlikely that ties will be banned simply because they are uncomfortable. (Jewish World Review. 6. 2002. <URL: http://www.jewishworldreview.com/1102/grimes110602.asp >)

Un caso similar de doble efecto interpretativo por yuxtaposición de secuencias literales, relacionadas en el plano compositivo, pero no homónimas, la hallamos con referencia a la UF alemana Ein Mann, ein Wort ('auf seine Aussage kann man sich verlassen', DUW), literalmente, 'un hombre, una palabra', pero que en su significado traslaticio se refiere más bien a la necesidad de cumplir lo que se promete o a lo que uno se compromete (cf. la UF española Lo prometido es deuda). Dicha unidad es parodiada en la frase "Ein Mann, ein Wort - eine Frau, ein Wörterbuch", como se puede observar en el siguente ejemplo:

Klischees, an denen Männer krampfhaft festhalten, um Frauen zu kontrollieren und um von ihren eigenen Schwächen abzulenken! Natürlich sind einige dieser Klischees veraltet, man hört sie kaum noch, aber Tatsache bleibt, daß sie von Männern stammen:

Frauen und Technik - zwei Welten begegnen sich

Frau am Steuer - ungeheuer

Eine Frau gehört am Herd

Ein Mann darf fremdgehen - eine Frau nie

Du bist doch nur eine Frau

Eine Frau hat zu gehorchen

Ein Mann ein Wort - eine Frau ein Wörterbuch

Sind wir Frauen etwa auch so gemein und haben Klischees ins Leben gerufen, die den Mann betreffen? <http://www.webarena.de/frauen.htm> 
La secuencia literal que sigue a la UF en cuestión ("Eine Frau, ein Wörterbuch") provoca la literalización de los componentes individuales de la UF: si el hombre, en el plano literal, es sólo una palabra, entoces la mujer debe ser cientos de ellas, todo un diccionario. El juego alusivo de los planos literal y figurativo hacen pues referencia a los prejuicios y los estudios de género, a los movimientos de liberación femenina, a la guerra de los sexos, etc., desde el punto de vista de la producción lingüística: la parquedad de palabras atribuida a los hombres contrasta con la supuesta "incontinencia verbal" de las mujeres. En este ejemplo se ha primado la interpretación literal, pero no sin antes haberse servido de la UF institucionalizada, desencadenándose la interpretación metafórica para descartarla inmediatamente.

El último ejemplo que vamos a analizar hace referencia a la UF española darse cabezazos contra la pared. Dicha unidad incorpora el gesto físico de golpear la cabeza, que normalmente sólo es de de dicto (estar extremadamente deseperado o furioso), pero pudiera serlo también de re (golpearse la cabeza de pura desesperación). En ausencia del gesto físico, esta UF denota la idea simbolizada por éste (cf. Corpas Pastor, 1996: 124-125). Véamos los efectos de la inserción de una secuencia homónima en el fragmento que sigue, extraído de una noticia de prensa sobre la solicitud presentada por el APA para que se expulse a un profesor de Deltebre (Tarragona), al cual acusan de un supuesto delito de maltrato psicológico a los alumnos. Dice así:

TARRAGONA. Los padres de alumnos de cuarto de ESO del colegio público Riomar de Deitebre han reclamado al departamento de Ensenyament que uno de los profesores del centro deje de dar clases a sus hijos... Según los padres, el maestro manipula y maltrata psicológica y físicamente a los niños de entre 8 y 9 años, llegando a actitudes insólitas, tal y como declaraba ayer una madre afectada. Según la mujer, sin ningún motivo aparente "este hombre se da cabezazos contra la pared y los niños no lo ven normal y tienen miedo". De los quince alumnos de cuarto de ESO, once no asisten a clase desde el lunes por fobia escolar y dos han cambiado de colegio. (El Mundo ${ }^{22}, 18-10-01$ )

La secuencia literal "se da cabezazos contra la pared" desencadena al mismo tiempo la interpretación metafórica de la UF homónima (darse cabezazos contra la pared). La referencia a la acción física de autolesionarse, golpeándose la cabeza contra la pared, nos dirige, en primera instancia, hacia el plano de re del contenido semántico de la UF institucionalizada. Dicha acción es cuanto menos inusual ("los niños no lo ven normal y tienen miedo"). Es difícil, pues, encontrarle una explicación, a menos que el sujeto presente un cuadro de desequilibrio mental (plano de dicto), provocado por la ansiedad que le pudiera provocar el dar clase o por otros motivos, quizá menos evidentes.

\section{Conclusión}

La variabilidad fraseológica, lejos de ser un fenómeno uniforme, constituye un hecho lingüístico que adopta diferentes formas explotando su capacidad en distintos niveles y con diferentes objetivos. Su naturaleza cambiante y su vertiente creativa la convierten en algunos casos y con relativa frecuencia en un fenómeno impredecible sometido a los caprichos y a la voluntad de los hablantes. Su categorización demuestra, además, que no se trata de 
un aspecto aislado y marginal dentro del universo fraseológico. Muy al contrario, la variabilidad constituye un aspecto central y fundamental en la descripción de las UFs constatado a través de la gran actividad que presentan sus categorías principales, a saber, las variantes y las modificaciones, en diferentes lenguas y registros textuales.

La distinción entre unas categorías y otras es, en ocasiones, una tarea complicada debido al carácter no discreto de la fraseología y sus manifestaciones. De ahí la necesidad de analizar la variabilidad dentro de su contexto. No en vano, en el estudio de ambos tipos de variabilidad, pero especialmente en el dedicado a las modificaciones, el contexto adquiere una importancia primordial, ya que representa una pieza clave y decisiva tanto en la localización como en la interpretación de las unidades.

Las unidades fraseológicas modificadas, por su parte y a diferencia de las variantes, actúan como potentes instrumentos generadores de cohesión textual, cuya fuerza supera incluso a la exhibida en la utilización de las UFs en su forma canónica o institucionalizada. Ello explica, pues, su alta frecuencia en determinados registros textuales.

La evolución de las nuevas tecnologías junto con el desarrollo de la lingüística del corpus hacen cada vez más evidente la importancia del estudio de las posiblidades de variación en la fraseología. Su recurrencia así lo demuestra. Por ello, a pesar de los estudios ya existentes acerca de algunas de las formas de la variabilidad en distintas lenguas, son aún necesarias las investigaciones que se ocupen de los aspectos menos analizados, sobre todo, con respecto a cada una de las esferas del sistema fraseológico en concreto y en el mayor número de lenguas posible.

\section{Referencias bibliográficas}

Academia Española de la Lengua (ed.) (1992): Diccionario de la lengua española. $21^{\text {a }}$ ed. Madrid, Espasa Calpe. [DRAE]

Baranov, A. N. y Dobrovol'skij, D. O. (1998): "Idiomaticidad e idiomatismos". En Luque Durán, J. y A. Pamies Bertrán (eds.) Léxico y fraseología. Granada: Granada Lingvistica y Método Ediciones, págs. 19-42.

Barz, I. (1986): "Probleme der phraseologischen Modifikation", Deutsch als Fremdsprache 23, 6, págs. 321-326.

Barz, I. (1992): "Phraseologische Varianten: Begriff und Probleme". En Földes, C. (ed.) Deutsche Phraseologie in Sprachsystem und Sprachverwendung. Viena, Praesens, págs. 25-47.

Burger, H. (1998): Phraseologie. Eine Einfiihrung am Beispiel des Deutschen. Berlin, Wrich Schmidt Verlag.

Burger, H. (2000): "Konzepte der «Variation» in der Phraseologie". En Häcki Buhofer, A. (ed.) Vom Umgang mit sprachlicher Variation. Soziolinguistik, Dialektologie, Methoden und Wissenschaftsgeschichte. Tübingen, Francke Verlag, págs. 35-51.

Burger, H., Buhofer, A. y Sialm, A. (1982): Handbuch der Phraseologie. Berlin y New York, Walter de Gruyter.

Černyševa, I. I. (1980): Feste Wortkomplexe des Deutschen in Sprache und Rede. Moscú, Vuisshay Shkola.

Corpas Pastor, G. (1994): Un estudio paralelo de los sistemas fraseológicos del inglés y del español. Tesis doctoral. Universidad Complutense de Madrid. (Edición en microfichas de la Universidad de Málaga. Servicio de Publicaciones e intercambio científico. 1995) 
Corpas Pastor, G. (1996): Manual de Fraseología Española. (Biblioteca Románica Hispánica. Manuales, 76). Madrid, Gredos.

Corpas Pastor, G. (1998a): "El uso de paremias en un corpus del español peninsular actual”. En Wotjak, G. (ed.) Estudios de fraseología y fraseografia del español actual. Veruert, Lingüística Iberoamericana, págs. 365-390. (Recogido en G. Corpas Pastor. 2003. Diez años de investigación en fraseología: análisis sintáctico-semánticos, contrastivos y traductológicos. Frankfurt am Main: Vervuert/Madrid: Iberoamericana. 83-107).

Corpas Pastor, G. (1998b): "Expresións fraseolóxicas e colocacións: clasificación”. En Ferro Ruibal, X. (ed.), págs. 31-61.

Corpas Pastor, G. (ed.) (2000): Las lenguas de Europa: Estudios de fraseología, fraseografia y traducción. Granada, Editoriai Comares.

Cowie, A. P.; Mackin, R. y McCaig, I. R. (1993): Oxford Dictionary of English Idioms. Oxford, Oxford University Press. [ODOEI]

Dobrovol'skij, D. (1988): Phraseologie als Objekt der Universalienlinguistik. Leipzig, VEB Verlag Enzyklopädie.

Dobrovol'skij, D. (1999): "Zu semantischen und pragmatischen Effekten kreativer Idiom-Modifikationen", Noveaux cahiers d'allemand 17 , págs. 363-374.

Drosdowski, G. (ed.) (1989): Duden Deutsches Universalwörterbuch. Mannheim, Wien y Zürich, Dudenverlag.

Fleischer, W. (1982): Phraseologie der deutschen Gegenwartssprache. Leipzig, VEB Bibliographisches Institut Leipzig.

Ferro Ruibal, X. (ed.) (1998): Actas do I Coloquio Galego de Fraseoloxía. Santiago de Compostela, Centro Ramçon Piñeiro, Xunta de Galicia.

Gläser, R. (1986): Phraseologie der englischen Sprache. Tübingen, Max Niemeyer.

Gréciano, G. (1982): "Forschungen zur Phraseologie", Zeitschrift für Germanistische Linguistik 11/2, págs. 232-241.

Götz, D., Haensch, G. y Wellmann, H. (2003): Langenscheidt Growörterbuch Deutsch als Fremdsprache. Berlín y Munich: Langenscheidt. [LGDAF]

Griesbach, H. y Schulz, N. (1981): Langenscheidt 1000 deutsche Redensarten. Berlín y Munich: Langenscheidt. [LDR]

Häusermann, J. (1977): Phraseologie, Hauptprobleme der deutschen Phraseologie auf der Basis sowjetischer Forschungsergebnisse. Tübingen, Niemeyer.

Hessky, R. y Ettinger, S. (1997): Deutsche Redewendungen. Ein Wörter- und Übungsbuch für Fortgeschrittene. Tübingen: Gunter Narr Verlag. [DR]

Koller, W. (1977): Redensarten. Linguistische Aspekte,Vorkommensanalysen, Sprachspiel. (Reihe Germanistische Linguistik 5) Tübingen, Niemeyer.

Martínez Marín, J. (1990): "Las expresiones fijas del español y la relación de antonimia", Actas del Congreso de la SGEL (XX Aniversario), (Tenerife, 2-6 de abril de 1990). Madrid, SGEL, págs. 622-629.

Mellado Blanco, C. (1997): "Usuelle Varianten der deutschen Phraseologismen", Forum, págs. $133-$ 143.

Mena Martínez, F. (2002): La desautomatización de las paremias inglesas por sustitución: un estudio cognitivo. Tesis doctoral (edición en CDRom). Murcia, Servicio de Publicaciones de la Universidad de Murcia.

Mena Martínez, F. (2003): "En torno al concepto de desautomatización de las unidades fraseológica: aspectos básicos", Tonos digital 5. http://www.um.es/tonosdigital/znum5/estudios/HEdesautomatizacion.htm (28-10-2003).

Mieder, W. y Litovkina, A. T. (2002): Twisted Wisdom: Modern Anti-Proverbs. DeProverbio.com. [TW] 
Moon, R. (1998): Fixed Expressions and Idioms in English: A Corpus-based Approach (Oxford Studies in Lexicology and Lexicography). Oxford, Oxford University Press.

Palm, Ch. (1995): Phraseologie. Eine Einführung. Tübingen: Gunter Narr Verlag.

Ruiz Gurillo, L. (1997): Aspectos de fraseología teórica española. Valencia, Universidad de Valencia.

Sabban, A. (1998): Okkasionelle Variationen sprachlicher Schematismen. Eine Analysefranzösischer und deutscher Presse- und Werbetexte, (Romanica Monacensia, 53) Tübingen, Günter Narr.

Salvador, V. y Piquer, A. (eds.) (2000): El discurs prefabricat. Estudis de fraseologia teórica i aplicada. (Actes de les Jornades de Fraseologia Comparada. Castelló de la Plana, 11-12 de noviembre de 1999). Castellón de la Plana, Universidad Jaume I.

Summers, D. (1989=1979): Longman Dictionary of English Idioms. Harlow, Londres: Longman. [LDOEI]

Thun, H. (1978): Probleme der Phraseologie. Untersuchungen zur wiederholten Rede mit Beispielen aus den Französischen, Italienischen, Spanischen und Romänischen, Beihefte zur Zeitschrift für Romanische Philologie 168. Tübingen, Max Niemeyer.

Yankah, K. (2000): "Proverb speaking as a creative process: The Akan of Ghana", DeProverbio.com, vol. 6, núm. 2, págs. 1-20: http://www.deproverbio.com/DPjournal/DP,6,2,00/YANKAH/ AKAN.html (28-10-2003).

Wotjak, B. (1992): Verbale Phraseolexeme in System und Text. Tübingen, Max Niemeyer.

Zuluaga Ospina, A. (1975): "La fijación fraseológica", Thesaurus, BICC (Boletín del Instituto Caro Y Cuervo) XXX núm.1, págs. 225-248.

Zuluaga Ospina, A. (1980): Introducción al estudio de las expresiones fijas. Frankfurt a.M., Verlag Peter D. Lang.

Zuluaga Ospina, A. (1997): "Sobre fraseoloxismos e fenómenos colindantes". En Ferro Ruibal, X. (ed.), págs. 15-30.

Zuluaga Ospina, A. (2001): "Análisis y traducción de unidades fraseológicas desautomatizadas", PhinN núm. 16, págs. 67-83. http://www.phin.de (28-10-2003). 